\title{
Cardiovascular biomarkers predict fragility fractures in older adults
}

\author{
Madeleine Johansson, ${ }^{1}$ Fabrizio Ricci, ${ }^{1,2}$ Giuseppe Di Martino, ${ }^{3}$ Cecilia Rogmark, ${ }^{1,4}$ \\ Richard Sutton, ${ }^{5}$ Viktor Hamrefors, ${ }^{1,6}$ Olle Melander, ${ }^{1,6}$ Artur Fedorowski ${ }^{1,7}$
}

- Additional material is published online only. To view please visit the journal online (http://dx.doi.org/10.1136/ heartjnl-2018-313741).

For numbered affiliations see end of article.

\section{Correspondence to} Dr Artur Fedorowski, Department of Cardiology, Skåne University Hospital, Malmö 214 28, Sweden; artur. fedorowski@med.lu.se

Received 14 June 2018 Revised 25 September 2018 Accepted 26 September 2018 Published Online First 15 October 2018

\section{Linked}

- http://dx.doi.org/10.1136/ heartjnl-2018-314099

\section{Check for updates}

(C) Author(s) (or their employer(s)) 2019. No commercial re-use. See rights and permissions. Published by BMJ.

To cite: Johansson M, Ricci F Di Martino G, et al. Heart 2019:105:449-454.

\section{ABSTRACT}

Objective To assess the role of four biomarkers of neuroendocrine activation and endothelial dysfunction in the longitudinal prediction of fragility fractures.

Methods We analysed a population-based prospective cohort of 5415 community-dwelling individuals (mean age, 68.9 \pm 6.2 years) enrolled in the Malmö Preventive Project followed during $8.1 \pm 2.9$ years, and investigated the longitudinal association between $\mathrm{C}$-terminal pro-arginine vasopressin (CT-proAVP), C-terminal endothelin-1 precursor fragment (CT-proET-1), the midregional fragments of pro-adrenomedullin (MR-proADM) and pro-atrial natriuretic peptide (MR-proANP), and incident vertebral, pelvic and extremity fractures.

Results Overall, 1030 (19.0\%) individuals suffered vertebral, pelvic or extremity fracture. They were older $(70.7 \pm 5.8$ vs $68.4 \pm 6.3$ years), more likely women $(46.9 \%$ vs $26.3 \%)$, had lower body mass index and diastolic blood pressure, were more often on antihypertensive treatment (44.1\% vs $38.4 \%$ ) and had more frequently history of fracture (16.3\% vs $8.1 \%)$. Higher levels of MR-proADM (adjusted HR (aHR) per 1 SD: $1.51,95 \% \mathrm{Cl} 1.01$ to $2.28, \mathrm{p}<0.001)$ and MRproANP (aHR: $1.23,95 \% \mathrm{Cl} 1.05$ to $1.45, \mathrm{p}<0.001$ ) were independently associated with increased risk of any fracture. The fracture risk increased linearly across MR-proANP quartiles. Individuals who were in the top quartile of all four biomarkers had a significant higher risk of fracture at any site (aHR: $2.32,95 \% \mathrm{Cl} 1.86$ to 2.91), vertebral fracture (aHR: $3.16,95 \% \mathrm{Cl} 1.97$ to 5.07) and femoral fracture (aHR: $2.35,95 \% \mathrm{Cl} 1.64$ to 3.36).

Conclusions Elevated levels of MR-proADM and MRproANP independently predict fragility fractures in older adults. In subjects with top quartile levels of all four biomarkers there is a twofold to threefold increase in risk of vertebral and femoral fractures.

\section{INTRODUCTION}

Fragility fractures are defined as fractures resulting from relatively mild trauma, such as falling from a standing height or low height of less than $1 \mathrm{~m}$, that healthy adult bones should otherwise be able to withstand. ${ }^{12}$ The main sites of fragility fractures are the thoracolumbar spine, the pelvis, hip and femur, proximal tibia, proximal humerus and the distal forearm. ${ }^{13}$ Fragility fractures account for over $90 \%$ of fractures in older people, ${ }^{4}$ and are often associated with traumatic falls. ${ }^{1}$

Among fall-predisposing factors, cardiovascular (CV) autonomic dysfunction may lead to circulatory instability, orthostatic intolerance, dizziness, syncope, unexplained falls and traumatic injuries, especially in older people. ${ }^{5-7} \mathrm{~A}$ previous population-based study demonstrated that CV autonomic dysfunction presenting as orthostatic hypotension $(\mathrm{OH})$, or elevated resting heart rate may predict common fragility fractures. ${ }^{8}$

In parallel, dysautonomic responses of CV system have been associated with alterations in circulating biomarkers of neuroendocrine activation and endothelial function such as copeptin, pro-endothelin-1, pro-adrenomedullin and pro-atrial natriuretic peptide. ${ }^{9}$ These biomarkers have been shown to be elevated in patients with symptomatic $\mathrm{OH}$ and cardiac syncope, although downregulated adrenomedullin and endothelin have also been found among patients with cardioinhibitory vasovagal reflex. ${ }^{10-13}$ Importantly, the increased levels of neuroendocrine activation biomarkers indicate not only risk of incident circulatory collapse and traumatic falls but may point to accompanying endothelial dysfunction and structural CV changes. ${ }^{14}$ Taken together, assessment of these biomarkers may be useful to detect covert disorders of $\mathrm{CV}$ system predisposing to orthostatic intolerance and syncope, as well as endothelial dysfunction and chronic inflammation, all of which indicate higher risk of fragility fractures in older people. ${ }^{615}$ Accordingly, our aim was prospectively to investigate the relationship between circulating levels of precursor fragments of four bioactive peptides: adrenomedullin, arginine-vasopressin, atrial-natriuretic-peptide and endothelin-1, and incidence of fragility fractures in community-dwelling older middle-aged subjects.

\section{METHODS}

\section{Study design and population}

The Malmö Preventive Project (MPP) is a large prospective population-based cohort study including 33346 citizens of Malmö, Sweden, who were enrolled in 1974-1992 (33\% women, mean age at enrolment 45 years; range $26-61$ years). ${ }^{16}$ A total of 18240 among approximately 25870 surviving participants (age, 68 years; participation rate, $70.5 \%$ ) attended a rescreening programme between 2002 and 2006, consisting of complete physical examination, self-administered questionnaire exploring medical history including level of physical activity (1-sedentary leisure time; $2-$ moderate exercise during leisure time; 3-regular exercise and training; 4-intensive or professional 
training) and fasting blood sampling. The details regarding the rescreening programme can be found elsewhere. ${ }^{17}$ The study complied with the Declaration of Helsinki, and all participants gave informed written consent.

\section{CV biomarkers}

We investigated four haemodynamically active CV biomarkers: C-terminal pro-arginine vasopressin (CT-proAVP; a stable peptide of the arginine vasopressin precursor), C-terminal endothelin-1 precursor fragment (CT-proET-1), the mid-regional fragments of pro-adrenomedullin (MR-proADM) and pro-atrial natriuretic peptide (MR-proANP) among randomly selected 5415 study participants. Plasma biomarkers were measured in fasting blood samples that had been frozen at $-80^{\circ} \mathrm{C}$ after collection during rescreening examination in years 2002-2006. Circulating levels of CT-proAVP, CT-proET-1, MR-proADM and MR-proANP were assessed using the following assays according to the manufacturer's instructions: Thermo Scientific B·R $\cdot A \cdot H \cdot M \cdot S$ CT-proAVP LIA, Thermo Scientific $\mathrm{B} \cdot \mathrm{R} \cdot \mathrm{A} \cdot \mathrm{H} \cdot \mathrm{M} \cdot \mathrm{S}$ CT-proET-1 KRYPTOR, Thermo Scientific $\mathrm{B} \cdot \mathrm{R} \cdot \mathrm{A} \cdot \mathrm{H} \cdot \mathrm{M} \cdot \mathrm{S}$ MR-proADM KRYPTOR and Thermo Scientific $\mathrm{B} \cdot \mathrm{R} \cdot \mathrm{A} \cdot \mathrm{H} \cdot \mathrm{M} \cdot \mathrm{S} \quad \mathrm{MR}$-proANP KRYPTOR (BRAHMS, Hennigsdorf, Germany). ${ }^{18} 19$

\section{Fracture data}

Fractures, which are of typical location for fragility injuries in elderly, served as the primary endpoint for the assessment of fall risk relative to the alterations in the assessed $\mathrm{CV}$ biomarkers. Fractures were defined according to International Classification of Diseases (ICD), 10th Revision. Information about fracture diagnoses (date and ICD-10 code) and the date of death have been retrieved from the Swedish National Hospital Discharge Register and the Swedish National Cause of Death Register covering the period from MPP rescreening date, that is, 21 January 2002 to 21 December 2006 until 31 December 2014. In this study, we included fractures affecting spine and thoracic cage (S12.x, S22.x, S32.x), arms, shoulders and hands (S42.x, S52.x and S62.x), pelvis (S32.x), as well as hips and femur (S72.x). In addition, codes signalling stress fractures or similar were also sought (M48.4, M84.3 and M96.6).

\section{Statistical analysis}

Group differences in continuous variables between fracture-positive and fracture-negative individuals were compared using one-way analysis of variance test, whereas group differences in categorical variables were compared using Pearson's $\mathrm{X}^{2}$ test. The distribution of all four biomarkers was right skewed and $\log$ transformation was performed. The log-transformed and standardised values of CT-proAVP, CT-proET-1, MR-proADM and MR-proANP were used as independent variables for Cox regression analysis. The multivariable adjusted model was built by entering age, gender, body mass index (BMI), systolic blood pressure (BP), heart rate, antihypertensive treatment, smoking, diabetes, level of physical activity and history of CV disease (myocardial infarction or stroke) and fracture as covariates. Further, the biomarkers were stratified into quartiles and used for Kaplan-Meier survival analysis. Then, the quartiles were used as an independent variable for Cox regression analysis in order to test the risk increment across the quartiles of tested biomarkers. The time variable was calculated as follow-up time between date of screening examination and date of first fracture, death or end of follow-up through 31 December 2014.
Table 1 Baseline characteristics of the study population $(n=5415)$

\begin{tabular}{llllc}
\hline Cherall & $\begin{array}{l}\text { Fracture } \\
\text { positive } \\
(\mathbf{n}=1030)\end{array}$ & $\begin{array}{l}\text { Fracture } \\
\text { negative } \\
(\mathrm{n}=4385)\end{array}$ & P values \\
\hline Age (years) & $68.9 \pm 6.2$ & $70.7 \pm 5.8$ & $68.4 \pm 6.3$ & $<0.001$ \\
\hline Sex (\% male) & 69.7 & 53.1 & 73.7 & $<0.001$ \\
\hline BMI (kg/m $\left.{ }^{2}\right)$ & $23.2 \pm 3.7$ & $22.7 \pm 3.9$ & $23.4 \pm 3.7$ & $<0.001$ \\
\hline Current smoker, $\mathrm{n}(\%)$ & $855(15.8)$ & $175(17.0)$ & $680(15.5)$ & 0.24 \\
\hline $\begin{array}{l}\text { Supine systolic BP } \\
\text { (mm Hg) }\end{array}$ & $145.7 \pm 20$ & $144.7 \pm 20.6$ & $146.0 \pm 20.5$ & 0.076 \\
\hline $\begin{array}{l}\text { Supine diastolic BP } \\
\text { (mm Hg) }\end{array}$ & $83.8 \pm 10.8$ & $82.9 \pm 10.7$ & $84.0 \pm 10.8$ & 0.003 \\
\hline Heart rate (bpm) & $70.9 \pm 12.3$ & $71.1 \pm 12.5$ & $70.9 \pm 12.2$ & 0.76 \\
\hline $\begin{array}{l}\text { Antihypertensive } \\
\text { treatment, } \mathrm{n}(\%)\end{array}$ & $2137(39.5)$ & $454(44.1)$ & $1683(38.4)$ & 0.001 \\
\hline Prevalent fracture, $\mathrm{n}(\%)$ & $524(9.7)$ & $168(16.3)$ & $356(8.1)$ & $<0.001$ \\
\hline Diabetes, $\mathrm{n}(\%)$ & $673(12.4 \%)$ & $134(13)$ & $539(12.3)$ & 0.48 \\
\hline CT-proAVP (pmol/L) & $9.6 \pm 11$ & $9.46 \pm 8.92$ & $9.66 \pm 11.9$ & 0.61 \\
\hline CT-proET (pmol/L) & $71.2 \pm 20$ & $73.9 \pm 22.1$ & $70.6 \pm 19.4$ & $<0.001$ \\
\hline MR-proADM (nmol/L) & $0.75 \pm 0.23$ & $0.79 \pm 0.27$ & $0.74 \pm 0.23$ & $<0.001$ \\
\hline MR-proANP (pmol/L) & $125.5 \pm 82.8$ & $138.1 \pm 88.3$ & $122.5 \pm 81.2$ & $<0.001$ \\
\hline Vals & & & & \\
\hline
\end{tabular}

Values are mean \pm SD unless otherwise indicated.

$\mathrm{BMI}$, body mass index; BP, blood pressure; CT-proAVP, copeptin; CT-proET-1, C-terminal endothelin-1; MR-proADM, mid-regional pro-adrenomedullin; MR-proANP, mid-regional pro-atrial natriuretic peptide.

The proportional hazards assumption was assessed plotting log-log survival curves of all predictors inserted in the model. If the curves remained approximately parallel, the proportional hazards assumption was satisfied. The Harrell's C-statistic was computed to evaluate overall adequacy of risk prediction procedures. We also tested presence of interaction between age, sex and antihypertensive therapy on the relationship between each $\mathrm{CV}$ biomarker and fracture risk. Additionally, we performed sensitivity analysis by fracture site, where in order to allow adequate multivariable adjustment we restricted the analysis to those subtypes with incidence $>100$ cases. Finally, we performed Fine and Gray proportional hazards models to estimate sub-HRs of $\mathrm{CV}$ biomarkers controlling for death as competing event. ${ }^{20}$ $\mathrm{C}$-statistic was also computed for competing risk models. All analyses were performed using SPSS Statistics V.24.0.0.1 (IBM) or SAS V.9.2. Only findings with a two-tailed $\mathrm{p}$ value $<0.05$ were considered significant.

\section{RESULTS}

During the follow-up period of $8.1 \pm 2.9$ years, a total of 1030 study participants $(19.0 \%)$ suffered either vertebral, thoracic, arm, pelvic, hip or femoral fractures. Patients with incident fracture were older, more likely women, had lower BMI, diastolic $\mathrm{BP}$, and had more often antihypertensive treatment and history of fracture compared with fracture-free participants (table 1).

In the fully adjusted Cox regression model (table 2), higher levels of MR-proADM (adjusted HR (aHR) per $1 \mathrm{SD}: 1.51$, 95\% CI 1.01 to $2.28, \mathrm{p}<0.001$ ) and MR-proANP (aHR: 1.23, $95 \%$ CI 1.05 to $1.45, \mathrm{p}<0.001$ ) were independently associated with increased risk of fragility fractures. Adjusted Cox model satisfied the proportional hazards assumption. Further, as shown in figure $1 \mathrm{~A}-\mathrm{D}$ and online supplementary table S5, the cumulative risk of incident fractures increased linearly from lowest to highest quartile of MR-proANP, with a significant trend across quartiles of CT-proET-1 and MR-proADM. As regards CT-proAVP, the fracture risk was similarly elevated in both the lowest and highest quartiles, compared with the two mid-quartiles; however, after full adjustment, the association with 
Table 2 Risk of incident fragility fractures in a population of 5415 older adults according to circulating levels of cardiovascular biomarkers, CTproAVP, CT-proET-1, MR-proADM and MR-proANP, in a Cox regression model

\begin{tabular}{|c|c|c|c|c|c|c|}
\hline \multirow[b]{2}{*}{ Biomarkers } & \multicolumn{2}{|l|}{ Unadjusted } & \multicolumn{2}{|c|}{ Adjusted for age and gender } & \multicolumn{2}{|l|}{ Fully adjusted* } \\
\hline & $\mathrm{HR}(95 \% \mathrm{Cl}) \dagger$ & C-statistic & $\mathrm{HR}(95 \% \mathrm{Cl}) \dagger$ & C-statistic & $\mathrm{HR}(95 \% \mathrm{Cl}) \dagger$ & C-statistic \\
\hline CT-proAVP & 0.99 (0.93 to 1.06$)$ & $0.504(0.432-0.611)$ & $1.06(0.99$ to 1.13$)$ & $0.641(0.543-0.766)$ & $1.04(0.98$ to 1.12$)$ & $0.666(0.555-0.787)$ \\
\hline CT-proET-1 & 1.30 (1.23 to 1.39$)$ & $0.574(0.448-0.698)$ & 1.19 (1.11 to 1.28$)$ & $0.647(0.544-0.770)$ & 1.15 (1.07 to 1.23$)$ & $0.668(0.556-0.789)$ \\
\hline MR-proADM & 1.37 (1.29 to 1.45$)$ & $0.577(0.450-0.693)$ & 1.21 (1.13 to 1.29$)$ & $0.649(0.544-0.772)$ & 1.21 (1.12 to 1.31$)$ & $0.670(0.559-0.795)$ \\
\hline MR-proANP & 1.34 (1.26 to 1.43$)$ & $0.580(0.455-0.698)$ & 1.19 (1.11 to 1.28$)$ & $0.646(0.543-0.770)$ & 1.19 (1.10 to 1.28$)$ & $0.669(0.556-0.793)$ \\
\hline
\end{tabular}

*Adjusted for age, gender, body mass index, systolic blood pressure, heart rate, antihypertensive treatment, diabetes, smoking, prevalent fractures, history of cardio/ cerebrovascular disease and self-reported physical activity.

THR is reported per 1 SD increase of log-transformed biomarker concentration.

CT-proAVP, copeptin; CT-proET-1, C-terminal endothelin-1; MR-proADM, mid-regional pro-adrenomedullin; MR-proANP, mid-regional pro-atrial natriuretic peptide.

CT-proAVP was no longer significant (Q1-Q4 vs Q2-Q3, aHR: $1.03,95 \%$ CI 0.95 to 1.10$)$. Higher level of CT-proET-1 independently predicted femoral fracture only (aHR: $1.17,95 \% \mathrm{CI}$ 1.01 to 1.35 ) (online supplementary table S8).

Individuals who were in the top quartile of all four biomarkers $(n=324)$ had a significantly higher risk of fracture at any site (aHR: 2.32, 95\% CI 1.86 to 2.91), vertebral fracture (aHR: $3.16,95 \% \mathrm{CI} 1.97$ to 5.07 ) and femoral fracture (aHR: 2.35, $95 \%$ CI 1.64 to 3.36 ) (table 3 ).
There were significant interactions between sex and all four CV biomarkers, between age and MR-proANP, and between antihypertensive treatment and CT-proAVP, for incident fracture risk (online supplementary tables S1-S4). In particular, the relationship with incident fractures appeared to be stronger for men with elevated levels of MR-proANP, MR-proADM and CT-proET-1, for women with elevated levels of CT-proAVP (online supplementary table S2), for older participants with elevated MR-proANP (online supplementary table S3) and for
A

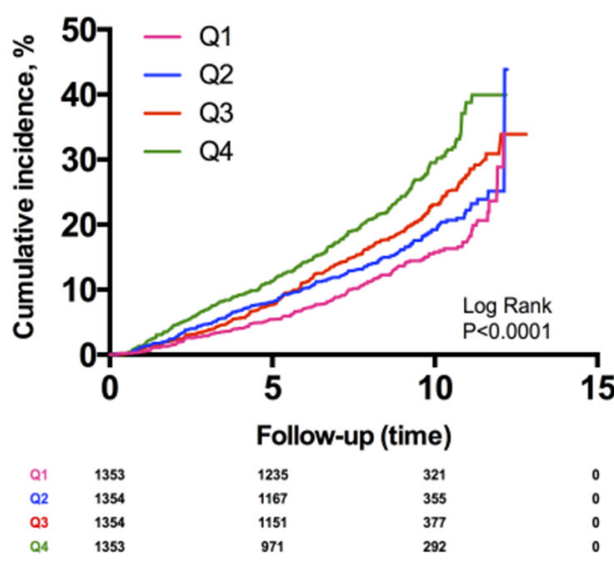

CT-proAVP

C

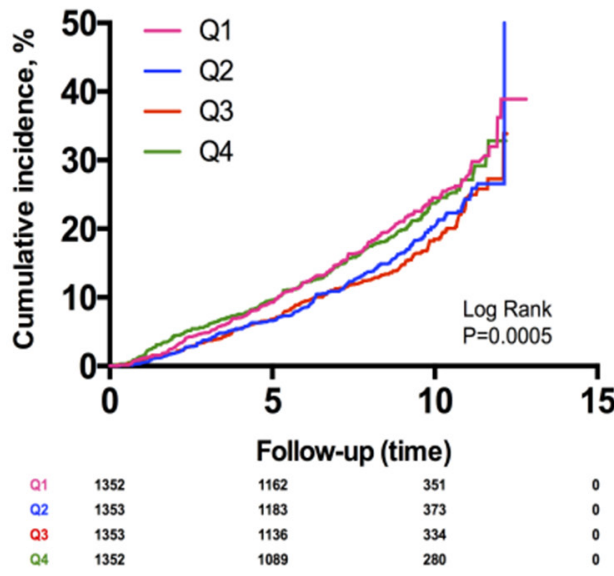

B

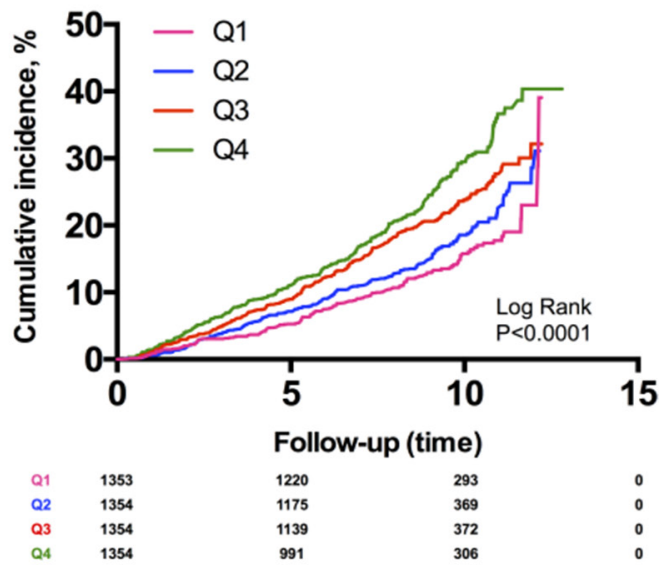

CT-proET-1

D

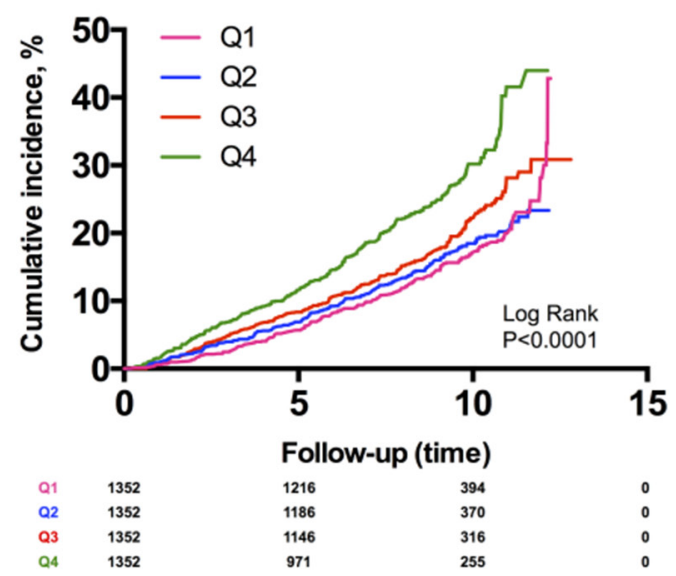

Figure 1 Kaplan-Meier curves for cumulative incidence of fragility fractures by quartiles of (A) MR-proADM, (B) MR-proANP, (C) CT-proAVP and (D) CT-proET-1. CT-proAVP, copeptin; CT-proET-1, C-terminal endothelin-1; MR-proADM, mid-regional pro-adrenomedullin; MR-proANP, mid-regional proatrial natriuretic peptide. 
Table 3 Risk of incident fragility fractures in patients in the fourth quartiles of cardiovascular biomarkers (CT-proAVP, CT-proET-1, MR-proADM and MR-proANP) in multivariable Cox regression model

\begin{tabular}{|c|c|c|c|c|c|c|}
\hline & \multicolumn{6}{|l|}{$\mathrm{HR}(95 \% \mathrm{Cl})$} \\
\hline & Fracture at any site & Vertebral $(n=104)$ & Upper $(n=118)$ & Forearm $(n=153)$ & Femoral $(n=312)$ & Tibial $(n=100)$ \\
\hline C-statistic $(95 \% \mathrm{Cl})$ & 0.656 (0.550 to 0.789$)$ & 0.659 (0.497 to 0.791$)$ & 0.641 (0.486 to 0.786$)$ & $0.645(0.490$ to 0.780$)$ & $0.648(0.501$ to 0.790$)$ & 0.641 ( 0.484 to 0.787$)$ \\
\hline $\begin{array}{l}\text { No biomarker in Q4 } \\
(\mathrm{n}=4067)\end{array}$ & Reference & Reference & Reference & Reference & Reference & Reference \\
\hline $\begin{array}{l}\text { One biomarker in Q4 } \\
(\mathrm{n}=518)\end{array}$ & 1.03 (0.67 to 1.10$)$ & 0.76 (0.33 to 1.51$)$ & 0.55 (0.13 to 1.02$)$ & 0.75 (0.43 to 1.33$)$ & 0.76 (0.46 to 1.23$)$ & $1.23(0.66$ to 2.30$)$ \\
\hline $\begin{array}{l}\text { Two biomarkers in Q4 } \\
(n=263)\end{array}$ & 1.36 (1.05 to 1.76$)$ & 2.16 (1.21 to 3.86$)$ & 1.20 (0.67 to 2.16$)$ & 1.47 (0.88 to 2.45$)$ & 1.54 (0.98 to 2.40$)$ & 1.01 (0.45 to 1.85$)$ \\
\hline $\begin{array}{l}\text { Three biomarkers in Q4 } \\
(n=247)\end{array}$ & $1.26(0.92$ to 1.64$)$ & 2.01 (1.07 to 3.39 ) & $1.13(0.63$ to 2.03$)$ & 0.78 (0.35 to 1.13$)$ & 0.98 (0.45 to 1.44$)$ & 0.95 (0.42 to 2.17$)$ \\
\hline $\begin{array}{l}\text { Four biomarkers in } \mathrm{Q4} \\
(\mathrm{n}=324)\end{array}$ & 2.32 (1.86 to 2.91$)$ & 3.16 (1.97 to 5.07$)$ & 1.18 (0.67 to 2.08$)$ & 0.83 (0.44 to 1.58$)$ & 2.35 (1.64 to 3.36$)$ & 1.21 (0.59 to 2.50$)$ \\
\hline
\end{tabular}

Adjusted for age, gender, body mass index, systolic blood pressure, heart rate, antihypertensive treatment, smoking, diabetes, prevalent fractures, history of cardio/ cerebrovascular disease and physical activity. Only fracture sites with incidence over 100 cases were analysed.

Q4, fourth quartile.

patients on antihypertensive treatment with elevated CT-proAVP (online supplementary table S4).

\section{DISCUSSION}

In this study, two biomarkers of neuroendocrine activation and endothelial dysfunction, MR-proADM and MR-proANP, independently predicted fragility fractures in older adults. Moreover, the combination of top quartiles of CT-proAVP, CT-proET-1, the MR-proADM and MR-proANP was independently associated with a twofold to threefold increase in risk of incident fractures, mostly at femoral and vertebral sites.

The assessed biomarkers have been previously found to predict adverse outcomes in patients with established CV disease such as coronary ischaemia or heart failure. ${ }^{21} 22$ Here, we have demonstrated that higher levels of these biomarkers may also predict fragility fractures in older people. Importantly, the strongest relationship was observed for MR-proADM and MR-proANP.

\section{Mid-regional fragment of pro-adrenomedullin}

MR-proADM is a precursor fragment of adrenomedullin with strong vasodilating properties. As previously reported, the plasma concentration of MR-proADM is significantly higher among patients with cardiac syncope (eg, primary cardiac arrhythmia and structural heart disease) as well as in syncope due to $\mathrm{OH} .{ }^{13}$ Increased MR-proADM levels were also found in patients with carotid sinus hypersensitivity, $\mathrm{OH}$ and unexplained syncope after initial evaluation, ${ }^{10}$ suggesting presence of either $\mathrm{CV}$ autonomic dysfunction or paroxysmal arrhythmia with or without underlying structural heart disease as the potential cause of fall trauma. However, adrenomedullin has other, apart from circulatory, properties such as regulation of insulin and bone metabolism. In particular, higher levels of adrenomedullin have been associated with osteoporosis, whereas treatment with adrenomedullin inhibitor has been related to increased bone density in animal models. ${ }^{23}$

\section{Mid-regional pro-atrial natriuretic peptide}

MR-proANP is a precursor fragment of vasodilating and diuretic hormone that controls vascular permeability and contractility, renin and aldosterone secretion, sympathetic nerve activity and renal tubular sodium transport. ${ }^{24}$ Elevated levels of MR-proANP have been linked to delayed $\mathrm{OH} .{ }^{9}$ Earlier studies have suggested that MR-proANP levels may serve as a marker of cardiac syncope. ${ }^{13}$ Sabatine et al showed that MR-proANP and
MR-proADM were, in fact, superior to $\mathrm{N}$-terminal pro-brain natriuretic peptide in predicting negative outcomes in chronic coronary disease. ${ }^{21}$ Thus, higher levels of ANP may predict susceptibility to $\mathrm{OH}$, cardiac syncope and, consequently, to increased fall risk. Although primarily active in the CV system, presence of ANP has been detected in human cartilage, ${ }^{25}$ suggesting that this neuropeptide may also be involved in bone and cartilage metabolism, pointing at other than circulatory mechanisms behind ANP-fragility fracture association.

The upregulation of CV hormones may indicate a susceptibility to develop manifest $\mathrm{CV}$ autonomic failure, $\mathrm{OH}$ and cerebral hypoperfusion, ${ }^{926}$ thus increasing the risk of traumatic falls. Interestingly, the higher likelihood of fracture was independently predicted by increasing levels of vasodilating biomarkers, MR-proADM and MR-proANP, but not by vasoconstrictors, CT-proAVP and CT-proET-1. A possible explanation could be that higher levels of circulating vasodilators decrease the ability to buffer wide fluctuations in BP and autoregulation of cerebral blood flow during orthostasis, shifting central blood volume to the periphery. Notably, combination of highest levels of all four biomarkers identified subjects with the highest likelihood of incident fracture, suggesting additive effect of information conferred by each of four biomarkers.

One might argue that higher levels of $\mathrm{CV}$ biomarkers could also predict $\mathrm{CV}$ events such as acute coronary events and cardiac arrhythmia, ${ }^{21}$ so, other than dysautonomic mechanisms may have been involved in traumatic falls. In fact, lower bone mineral density, implicating higher susceptibility to fragility fractures, is associated with increased coronary calcification index and risk of coronary disease. ${ }^{27}$ Although the design of our study does not allow detailed analysis of all fracture scenarios, it is estimated that primary cardiac arrhythmias are responsible for about 10\%-15\% cases of unexplained syncope, and, probably, a similar proportion of unexplained falls. ${ }^{28}$ Thus, the assessed biomarkers may herald risk of circulatory collapse due to autonomic dysfunction and primary heart disease, especially in regard to increased MR-proANP. ${ }^{13}$ However, syncope due to autonomic disorders, reflex syncope and $\mathrm{OH}$ are much more frequent than primary cardiac disease. ${ }^{28}$

To give a balanced view of the reported association between increased levels of haemodynamically active hormones and the incidence of fragility fractures, we might consider an alternative scenario where the biomarkers of endothelial dysfunction indicate microvascular disorders in bone tissue leading to 
bone fragility. The pathophysiological mechanisms underlying bone fragility in older people are complex and both reduced resistance to stress and increased risk of falling contribute to fragility fractures, as observed in diabetes mellitus. ${ }^{29}$ Accordingly, CV biomarkers may confer information about both global neuroendocrine hyperactivation and microvascular endothelial dysfunction and point to a synergic effect of $\mathrm{CV}$ imbalance and circulatory-mediated fall risk associated with negative alterations in bone structure.

In a subgroup analysis, the observed relations of MR-proADM and MR-proANP with fragility fractures were stronger in men, who were, however, over-represented in the study sample $(\approx 70 \%)$. Another potential explanation could be an important impact of menopause on bone fragility in women, a factor that is not present in men. Consequently, haemodynamic and metabolic pathological mechanisms signalled by increased levels of CV biomarkers may play a more important role in older middleaged men than in postmenopausal women.

As the population of older individuals increases, the absolute number of fragility fractures is also expected to rise. ${ }^{30}{ }^{31}$ Lagi et al showed that almost $30 \%$ of older patients without memory deficit had difficulties recalling a minor fall after 3 months. ${ }^{12}$ Considering that nearly $50 \%$ of cases are not witnessed, markers that could exclude or confirm orthostatic instability and syncope would be of great value. It might be even more important to identify older adults who demonstrate higher risk of fragility fractures and apply CV biomarkers for risk stratification and possible prophylactic strategies such as more restrictive use of fall-risk-increasing $\operatorname{drugs}^{32}$ and home adaptations.

\section{Strengths and limitations}

The principal strengths of this work were the large study population and extensive follow-up time, as well as reliable data registers. Nevertheless, we recognise some limitations that should be addressed. First, we acknowledge the lack of prospective biomarker assessment during follow-up as well as lack of important pieces of information such as calcium and phosphorus metabolism, bone mineral density, vitamin D levels and use of medications-other than antihypertensive drugs-with potential adverse effects on orthostatic stability, alertness, balance and neuroendocrine activation. Second, although we performed adjustments for multiple potential confounders, we cannot exclude possibility of residual confounding not accounted for by available covariates. Thus, the differences in biomarker levels might be also due to the fact that fragility fracture-prone individuals are of poorer general health compared with the rest of cohort. Third, in competing risk analysis controlling for death, MR-proADM and MR-proANP lost their significant association with fragility fractures, while higher levels of MR-proANP showed non-significant tendency towards increased fracture risk in fully adjusted model (aHR: $1.23,95 \%$ CI 0.99 to 1.38 , $\mathrm{p}=0.06$ ) (online supplementary tables $\mathrm{S} 6$ and S7). Fourth, register-derived fracture codes may have included a smaller number of high-energy fractures but they were presumably evenly distributed among the groups. Finally, we hypothesised that the majority of fragility fractures resulted from a fall due to diminished cerebral perfusion, although low-energy fractures may occur in other situations such as tripping or slipping.

\section{CONCLUSIONS}

Higher levels of MR-proADM and MR-proANP predict incident fragility fractures in older adults. In subjects with top quartile levels of the four biomarkers there was a twofold to threefold increase in risk of incident fracture, mostly involving vertebral and femoral sites. Further research is needed to assess the utility of $\mathrm{CV}$ biomarkers in understanding and prevention of fragility fractures.

\section{Key messages}

What is already known on this subject?

- Both cardiovascular autonomic disorders and endothelial dysfunction are associated with higher risk of fragility fractures among older subjects.

\section{What might this study add?}

- Elevated levels of biomarkers of neuroendocrine activation and endothelial dysfunction: mid-regional fragments of pro-adrenomedullin and pro-atrial natriuretic peptide independently predict fragility fractures in older adults. Moreover, the combination of top quartiles of these and two other biomarkers, C-terminal pro-endothelin-1 and pro-arginine vasopressin, indicated a twofold to threefold increase in risk of incident fracture, mostly involving vertebral and femoral sites.

\section{How might this impact on clinical practice?}

- Biomarkers of neuroendocrine activation and endothelial dysfunction should be considered for the development of predictive models aimed at early identification of individuals at high fracture risk who might benefit most from effective prevention strategies, such as more restrictive use of antihypertensive and psychopharmacological drugs as well as home adaptations.

\section{Author affiliations}

'Department of Clinical Sciences, Faculty of Medicine, Clinical Research Center, Lund University, Malmö, Sweden

${ }^{2}$ Department of Neuroscience, Imaging and Clinical Sciences, Institute for Advanced Biomedical Technologies, 'G d'Annunzio' University, Chieti, Italy

${ }^{3}$ Department of Medicine and Ageing Sciences, School of Hygiene and Preventive

Medicine, 'G d'Annunzio' University, Chieti, Italy

${ }^{4}$ Department of Orthopedics, Skåne University Hospital, Malmö, Sweden

${ }^{5}$ National Heart and Lung Institute, Imperial College London, London, UK

${ }^{6}$ Department of Internal Medicine, Skåne University Hospital, Malmö, Sweden

${ }^{7}$ Department of Cardiology, Skåne University Hospital, Malmö, Sweden

Funding This work was supported by grants from the Swedish Medical Research Council, the Swedish Heart and Lung Foundation, the Medical Faculty of Lund University, Malmö University Hospital, the Albert Påhlsson Research Foundation, the Crafoord Foundation, the Ernhold Lundströms Research Foundation, the Region Skane, the Hulda and Conrad Mossfelt Foundation, the King Gustaf $V$ and Queen Victoria Foundation, The Wallenberg Foundation and the Lennart Hanssons Memorial Fund.

Competing interests $\mathrm{AF}$ reports personal fees from Cardiome and a patent Thermo Fisher pending outside the submitted work. RS reports personal fees and other from Medtronic, Abbott Laboratories outside the submitted work; performs consultancy for Medtronic; is a member of the speakers' bureau of Abbott Laboratories; and is a shareholder in Boston Scientific, Edwards Lifesciences and AstraZeneca.

Patient consent Not required.

Ethics approval Regional Ethical Review Board of Lund University, Sweden.

Provenance and peer review Not commissioned; externally peer reviewed.

Data sharing statement No additional data are available.

\section{REFERENCES}

1 Cooper $C$. The crippling consequences of fractures and their impact on quality of life Am J Med 1997;103:S12-S19. discussion 7S-9S. 
2 Orcel P, Funck-Brentano T. Medical management following an osteoporotic fracture. Orthop Traumatol Surg Res 2011;97:860-9.

3 Johnell O, Kanis J. Epidemiology of osteoporotic fractures. Osteoporos Int 2005;16(SO 2):S3-7.

4 Court-Brown CM, McQueen MM. Global forum: fractures in the elderly. J Bone Joint Surg Am 2016:98:e36.

5 Finucane C, O'Connell MD, Donoghue 0, et al. Impaired orthostatic blood pressure recovery is associated with unexplained and injurious falls. J Am Geriatr Soc 2017;65:474-82.

6 Cronin H, Kenny RA. Cardiac causes for falls and their treatment. Clin Geriatr Med 2010;26:539-67.

7 Gangavati A, Hajjar I, Quach L, et al. Hypertension, orthostatic hypotension, and the risk of falls in a community-dwelling elderly population: the maintenance of balance, independent living, intellect, and zest in the elderly of Boston study. J Am Geriatr Soc 2011;59:383-9.

8 Hamrefors V, Härstedt M, Holmberg A, et al. Orthostatic hypotension and elevated resting heart rate predict low-energy fractures in the population: the malmö preventive project. PLoS One 2016;11:e0154249.

9 Nilsson D, Sutton R, Tas W, et al. Orthostatic changes in hemodynamics and cardiovascular biomarkers in dysautonomic patients. PLoS One 2015;10:e0128962.

10 Fedorowski A, Burri P, Struck J, et al. Novel cardiovascular biomarkers in unexplained syncopal attacks: the SYSTEMA cohort. J Intern Med 2013;273:359-67.

11 Hamrefors V, Nilsson D, Melander O, et al. Low adrenomedullin and endothelin-1 predict cardioinhibitory response during vasovagal reflex in adults over 40 years of age. Circulation 2017:10:e005585.

12 Lagi A, Cuomo A, Veneziani F, et al. Copeptin: a blood test marker of syncope. Int J Clin Pract 2013:67:512-5.

13 Badertscher P, Nestelberger T, de Lavallaz JDF, et al. Prohormones in the early diagnosis of cardiac syncope. J Am Heart Assoc 2017;6:6.

14 Richards AM. Future biomarkers in cardiology: my favourites. European Heart Journal Supplements 2018;20:G37-G44.

15 Augustin HG, Koh GY. Organotypic vasculature: from descriptive heterogeneity to functional pathophysiology. Science 2017;357:eaal2379.

16 Fedorowski A, Stavenow L, Hedblad B, et al. Orthostatic hypotension predicts all-cause mortality and coronary events in middle-aged individuals (The Malmo Preventive Project). Eur Heart J 2010;31:85-91.

17 Fava C, Sjögren M, Montagnana M, et al. Prediction of blood pressure changes over time and incidence of hypertension by a genetic risk score in Swedes. Hypertension 2013:61:319-26.
18 Fenske W, Störk S, Blechschmidt A, et al. Copeptin in the differential diagnosis of hyponatremia. J Clin Endocrinol Metab 2009;94:123-9.

19 Caruhel P, Mazier C, Kunde J, et al. Homogeneous time-resolved fluoroimmunoassay for the measurement of midregional proadrenomedullin in plasma on the fully automated system B.R.A.H.M.S KRYPTOR. Clin Biochem 2009:42:725-8.

20 Fine JP, Gray RJ. A proportional hazards model for the subdistribution of a competing risk. J Am Stat Assoc 1999;94:496-509.

21 Sabatine MS, Morrow DA, de Lemos JA, et al. Evaluation of multiple biomarkers of cardiovascular stress for risk prediction and guiding medical therapy in patients with stable coronary disease. Circulation 2012;125:233-40.

22 von Haehling S, Filippatos GS, Papassotiriou J, et al. Mid-regional pro-adrenomedullin as a novel predictor of mortality in patients with chronic heart failure. Eur J Heart Fail 2010;12:484-91.

23 Martínez-Herrero S, Larrayoz IM, Ochoa-Callejero L, et al. Prevention of bone loss in a model of postmenopausal osteoporosis through adrenomedullin inhibition. Front Physiol 2016;7:280

24 Bie P. Natriuretic peptides and normal body fluid regulation. Compr Physiol 2018;8:1211-49

25 Lipari L, Gerbino A, Lipari A, et al. Atrial natriuretic Peptide expression in human articular cartilage. J Biol Regul Homeost Agents 2015;29:235-8.

26 Bachus $\mathrm{E}, \mathrm{Holm} \mathrm{H}$, Hamrefors $\mathrm{V}$, et al. Monitoring of cerebral oximetry during headup tilt test in adults with history of syncope and orthostatic intolerance. Europace 2018;20:1535-42.

27 Ahmadi N, Mao SS, Hajsadeghi F, et al. The relation of low levels of bone mineral density with coronary artery calcium and mortality. Osteoporos Int 2018;29:1609-16.

28 Brignole M, Moya A, de Lange FJ, et al. 2018 ESC Guidelines for the diagnosis and management of syncope. Eur Heart J 2018;39:1883-948.

29 Napoli N, Chandran M, Pierroz DD, et al. Mechanisms of diabetes mellitus-induced bone fragility. Nat Rev Endocrinol 2017;13:208-19.

30 Mathew SA, Gane E, Heesch KC, et al. Risk factors for hospital re-presentation among older adults following fragility fractures: a systematic review and meta-analysis. $B M C$ Med 2016;14:136.

31 Svedbom A, Hernlund E, Ivergård M, et al. Osteoporosis in the European Union: a compendium of country-specific reports. Arch Osteoporos 2013;8:137.

32 Ernsth Bravell M, Westerlind B, Midlöv P, et al. How to assess frailty and the need for care? Report from the Study of Health and Drugs in the Elderly (SHADES) in community dwellings in Sweden. Arch Gerontol Geriatr 2011:53:40-5. 